\title{
Circulating Innate Lymphoid Cells Exhibit Distinctive Distribution During Normal Pregnancy
}

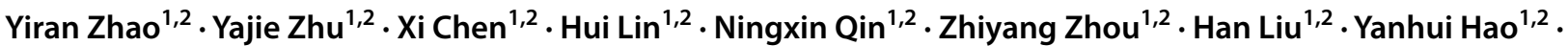

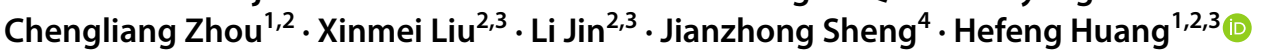

Received: 24 February 2021 / Accepted: 14 December 2021 / Published online: 5 January 2022

(c) The Author(s) 2022

\begin{abstract}
Over the past decades, the investigation of innate lymphoid cells (ILCs) has revealed their significance in successful pregnancy. Sex hormones, such as estradiol and progesterone, show specific changes during pregnancy and modulate both adaptive and innate immune systems. ILC subset distribution in peripheral blood of pregnant women and its potential association with sex hormone levels have not been well revealed. Peripheral blood was obtained from healthy non-pregnant, early-pregnant, and late-pregnant women. Radioimmunoassay was performed to measure plasma estradiol and progesterone levels. The levels of type 1 ILCs (ILC1s), type 2 ILCs (ILC2s), type 3 ILCs (ILC3s), and total ILCs as well as estrogen and progesterone receptors of ILC2s in peripheral blood were analyzed using flow cytometry. The proportion of total ILCs and distribution of ILC subsets in peripheral blood changed dynamically during pregnancy. Compared to non-pregnant women, late-pregnant women displayed significantly higher proportion of circulating ILCs, among which ILC2s accounted for the majority in late-pregnant women while a smaller part in others, and ILC3s displayed the opposite. Plasma estradiol and progesterone levels elevated while pregnancy proceeded and the expression of their receptors in ILC2s increased consisted with the proportion of circulating ILC2s. Our work first observed the existence of progesterone receptors in human circulating ILC2s and revealed the distribution pattern of circulating ILC subsets and their interrelation with plasma sex hormone levels during pregnancy. Our results suggested that the estradiol and progesterone levels might partly influence the distribution of circulating ILC subsets and implied the interplay between circulating ILCs and pregnancy.
\end{abstract}

Keywords Innate lymphoid cells $\cdot$ Innate immunity $\cdot$ Pregnancy $\cdot$ Sex hormones $\cdot$ Estradiol $\cdot$ Progesterone

\section{Introduction}

Yiran Zhao and Yajie Zhu contributed equally to this work.

Hefeng Huang

huanghefg@sjtu.edu.cn

1 The International Peace Maternity \& Child Health Hospital, School of Medicine, Shanghai Jiao Tong University, Shanghai 200030, China

2 Shanghai Key Laboratory of Embryo Original Diseases, Shanghai 200030, China

3 Hospital of Obstetrics and Gynecology, Fudan University, Shanghai 200010, China

4 Department of Pathology and Pathophysiology, School of Medicine, Zhejiang University, Zhejiang 310058, China
Successful pregnancy relies on the sophisticated balance of the immune system which maintains tolerance to the semiallogeneic fetus, while sustains innate and adaptability to meet challenges [1-3]. Recent studies have shown that $\mathrm{T}$ cells and their subgroups play a key role in preserving fetal development [4-7]. While the adaptive immune system plays an important role, the innate immune system also matters [1, 8-12]. Innate lymphoid cells (ILCs) are a developing family of innate immune cells lacking lineage markers for $\mathrm{T}$ cells, B cells, myeloid and dendritic cells, monocytes and macrophages, mast cells, and stem cells and mirror the functions of T cells. Natural killer (NK) cells were the only known ILCs for much of past years; however, several new ILC populations have been identified recently. Identical to lymphocytes, ILCs develop from the common lymphoid 
progenitor, but distinct transcription factors suppress the lymphocyte fates and lead to the generation of the different subsets of ILCs. According to the functions parallel to those of T cells, ILCs can be generally classified into two types: helper type ILCs and cytotoxic ILCs. Helper type ILCs include type 1 ILCs (ILC1s), type 2 ILCs (ILC2s), and type 3 ILCs (ILC3s), which are respectively the innate counterparts of $\mathrm{CD} 4^{+} \mathrm{T}$ helper (Th) 1, Th2, and Th17 cells. As a conventional group of innate cells, NK cells are termed as cytotoxic ILCs recently, mirror the functions of CD8 ${ }^{+}$ cytotoxic T cells $[13,14]$. Over the last decade, ILCs have been identified not only in human mucosal tissues, but also non-mucosal tissues, such as peripheral blood [15].

More recently, the significance of ILCs in successful pregnancy has been revealed [12, 16-20]. ILCs have been identified in maternal-fetal compartments in early weeks of pregnancy in human as well as mice [8], among which, NK cells are the most conspicuous during the first trimester of pregnancy in human decidua [21], while ILC2s were just a small proportion [12]. In the process of pregnancy, NK cells start to decline and ILC2s gradually become the most abundant subsets in human decidua of late pregnancy [19]. Moreover, ILC2 is the most abundant ILC subset in murine uterus [22], indicating their underline effect in maintaining normal pregnancy. The dynamic changes of ILC subsets in human decidua and murine uterus suggest an important role of ILCs in maintaining healthy pregnancy, and an abnormal increasing of ILC subsets may lead to a pathologic pregnancy, such as preterm labor [19, 23].

Hormones are also necessary for maintaining the adequate environment for successful pregnancy. Particularly, sex hormones are of great importance, the most studied are progesterone and estrogens [24-29]. Estradiol (E2), the predominant and most biologically active form, belongs together with estrone (E1) and estriol (E3) to estrogens [30]. During three trimesters of pregnancy, progesterone and estradiol levels elevate as pregnancy progresses, which adapt to the physiological changes [24, 25]. Progesterone and estradiol have been reported to modulate both adaptive and innate immune cells in human and mice, such as T cells, B cells, macrophages, dendritic cells, and NK cells [31-41]. As previously described, estradiol has an estrogen receptor $\alpha$ $(\mathrm{ER} \alpha)$-selective agonist potency [42], which mainly exists in the nucleus, and can regulate ILC2 accumulation in murine uterus as well as modulate expression of a series of genes through highly expressed ER $\alpha$ [43].

Both helper type ILCs and NK cells as well as sex hormones, as mentioned above, are of great importance for healthy pregnancy. Compared to NK cells, the variation of circulating helper type ILCs during normal pregnancy remains unrevealed. Our study aimed to explore the dynamic changes of helper type ILC subsets before and during normal pregnancy, and whether plasma sex hormone levels correlate with ILC distribution in human peripheral blood. Using flow cytometry, we investigated the proportion and absolute counts of circulating helper type ILC subsets in non-pregnant and pregnant women as well as their correlation with increasing plasma progesterone and estradiol levels. Generally, our study is the first to discover the variation of circulating helper type ILC distribution in different gestation and observed the expression of progesterone receptor in human circulating ILC2s. Our work provides new insights into the potential mechanisms of sex hormones influencing ILCs in peripheral blood and indicates the physiological functions of circulating ILCs during pregnancy.

\section{Methods and Materials}

\section{Study Population and Sampling}

All subjects were Chinese pregnant women from The International Peace Maternity and Child Health Hospital (Shanghai, China) between April 2020 and January 2021. Twentyfive non-pregnant women, 25 early-pregnant (6-12 weeks) women, and 25 late-pregnant (29-42 weeks) women were all from outpatient department. Ethical approval for the study was obtained from the Ethics Review Committee/Institutional Review Board (GKLW 2017-01). Written informed consent was obtained from each participant. Only healthy women who were 25-35 years old were included, and pregnant women were all with a normal pregnancy. Previous pregnancies and deliveries must have been uncomplicated. Exclusion criteria were women who had a history of preeclampsia or gestational hypertension, diabetes, thyroid disease, anemia, intrahepatic cholestasis of pregnancy, spontaneous abortion, or preterm delivery. Gestational age was estimated by the crown-rump length, which measured by an ultrasound scan. Samples of peripheral blood from each group were included in the study, and there were no overlaps among three groups.

\section{Isolation of Plasma and Peripheral Blood Mononuclear Cells (PBMCs)}

Venous blood samples of non-pregnant and pregnant women were collected using routine tubes, respectively. After centrifuged at $4{ }^{\circ} \mathrm{C}$ for $15 \mathrm{~min}$ at $2000 \mathrm{~g}$, plasma was obtained and stored at $-80{ }^{\circ} \mathrm{C}$ for the measurement of estradiol and progesterone. Using Ficoll-Hypaque density gradient centrifugation at $20^{\circ} \mathrm{C}$ for $30 \mathrm{~min}$ at $900 \mathrm{~g}$, peripheral blood mononuclear cells (PBMCs) were obtained from $1 \mathrm{ml}$ peripheral blood and counted by Cellometer Auto 1000 (Nexcelom Bioscience, Lawrence, USA), then followed by analyses of 
ILC subsets using flow cytometry. Analyses were performed on fresh samples.

\section{Flow Cytometry}

To identify ILC subsets, the following antibodies to human proteins were stained with PBMCs: FITC-conjugated antiCD5 (UCTH2), anti-CD16 (3G8), anti-CD56 (NCAM16.2), anti-CD11c (B-ly6), anti-CD34 (581), anti-TCRa $\beta$ (T10B9.1A-31), APC-Cy7-conjugated anti-CD45 (2D1), BV421-conjugated anti-CRTH2 CD294 (CRTH2; BM16), APC- or APC-R700-conjugated anti-CD117 (C-Kit; YB5. B8), and R Phycoerythrin-Cyanine 7 (PE-Cy7)-conjugated anti-CD127 (HIL-7R-M21; all from BD Biosciences, Mountain View, USA); FITC-conjugated anti-CD3 $\varepsilon$ (HIT3a), antiCD4 (RPA-T4), and anti-CD11b (M1/70; all from BioLegend, San Diego, USA); FITC-conjugated anti-CD14 (61D3), anti-CD19 (HIB19), anti-CD123 (6H6), anti-TCR $\gamma \delta$ (B1.1), and anti-FceRIa (AER-37 (CRA1); all from eBioscience, San Diego, USA).

For measurement of intranuclear estrogen and progesterone receptors, PBMCs were isolated directly ex vivo, firstly stained with antibodies to surface antigens, fixed and permeabilized using Foxp3/Transcription Factor Staining Buffer Set (eBioscience, San Diego, USA) according to the manufacturer's instructions, and secondly stained with phycoerythrin (PE)-conjugated anti-ER $\alpha$ (ab209288, Abcam, Cambridge, UK) and Alexa Fluor 647-conjugated antiprogesterone receptor (D8Q2J, Cell Signaling Technology, Danvers, USA).

Samples were acquired on BD FACSCelesta flow cytometer instrument (BD, Franklin Lakes, USA) and analyzed using FlowJo software, version 10.7.0 (BD, Franklin Lakes, USA).

\section{Radioimmunoassay (RIA)}

The plasma concentrations of estradiol and progesterone in non-pregnant, early-pregnant, and late-pregnant women were measured by radioimmunoassay (RIA) using XH6080 radio-immune analyzer (Xi' an Nuclear Instrument Factory, $\mathrm{Xi}$ ' an, China) and following the manufacturer's instructions (Beijing North Institute of Biotechnology Co., Ltd).

\section{Statistical Analysis}

Statistical analyses were performed with GraphPad Prism software, version 8.4.3 (GraphPad Software Inc., CA, USA). The Kruskal-Wallis tests, one-way ANOVA, and chi-square tests were employed for comparisons among three study groups, and Mann-Whitney test was used for comparisons between early-pregnant and late-pregnant groups. To analyze the correlations between estradiol as well as progesterone levels and ILC2 proportion, Spearman's correlation analysis was performed. $P$-values less than $0.05(P<0.05)$ were considered significant. Graphs were plotted using GraphPad Prism software, version 8.4.3 (GraphPad Software Inc., CA, USA).

\section{Results}

\section{Patient Characteristics}

Seventy-five healthy women who were 25-35 years old were included, of which 25 were non-pregnant, 25 were earlypregnant, and 25 were late-pregnant (Table 1). No significant difference was noted in age, pre-pregnancy body mass index (BMI), and times of previous induced abortions among three study groups. Since pregnant women employed periodical inspection where blood sample were collected, the gestational age of early-pregnant and late-pregnant women was relatively concentrated in a certain interval.

\section{Late Pregnancy Displays the Highest-Circulating ILC Proportion in Human Peripheral Blood}

ILCs are a discrete population of $\mathrm{CD} 5^{+}$cells defined as lineage-negative $\left(\mathrm{Lin}^{-}\right)$cells with the expression of the a-chain of the IL-7 receptor (CD127) [19, 44]. As previously described, a combination of Lin markers, comprised of CD3e, CD4, CD5, CD14, CD16, CD19, CD56, CD11c, CD11b, CD34, CD123, TCRa $\beta$, TCR $\gamma \delta$, and FceRIa, was used in this study [44-46]. The gating strategy used to identify circulating ILC subsets is shown in Fig. 1a.

To explore the physiological changes of circulating ILCs in non-pregnant and pregnant women of different trimesters, we first detected the percentages of total ILCs within CD $45^{+}$ cells in peripheral blood. We found that the level of total ILCs was significantly higher in late-pregnant women than the non-pregnant group $(P=0.0053)$, while it was comparable to early-pregnant women $(P=0.6975)$ (Fig. 1b). Then we analyzed the absolute counts of ILCs per milliliter of blood in three study groups. No statistically significant difference in ILC absolute counts was found among three study groups $(P>0.05)$ (Fig. 1c).

\section{ILC Subsets Show Distinctive Distribution in Peripheral Blood Before and During Pregnancy}

To further determine which group of circulating ILCs has changed during pregnancy, we conducted an analysis of the proportion and phenotypes of ILC subsets. ILC1s and ILC3s were respectively defined as $\mathrm{CD}_{117^{-} \mathrm{CRTH}}{ }^{-}$and $\mathrm{CD} 117^{+} \mathrm{CRTH} 2^{-}$cells, and $\mathrm{CRTH} 2^{+}$cells were considered as ILC2s [47] (Fig. 1a). 
Table 1 Participant characteristics

\begin{tabular}{|c|c|c|c|c|}
\hline Characteristic & Non-pregnant & Early-pregnant & Late-pregnant & $P$ value \\
\hline$N$ & 25 & 25 & 25 & \\
\hline Age, mean $(S D)$, years & $30(3)$ & $29.72(2.31)$ & $30.36(2.66)$ & 0.6990 \\
\hline Pre-pregnancy BMI, mean $(S D), \mathrm{kg} / \mathrm{m}^{2}$ & $20.12(1.32)$ & $20.21(1.48)$ & $20.39(2.20)$ & 0.8511 \\
\hline Gravidity, $n(\%)$ & & & & $<0.001 * * *$ \\
\hline 0 & $7(28)$ & $0(0)$ & $0(0)$ & \\
\hline 1 & $8(32)$ & $4(16)$ & $15(60)$ & \\
\hline$\geq 2$ & $10(40)$ & $21(84)$ & $10(40)$ & \\
\hline Parity, $n(\%)$ & & & & $0.0176^{*}$ \\
\hline 0 & $15(60)$ & $8(32)$ & $19(76)$ & \\
\hline 1 & $4(16)$ & $9(36)$ & $5(20)$ & \\
\hline$\geq 2$ & $6(24)$ & $8(32)$ & $1(4)$ & \\
\hline Previous induced abortions, $n(\%)$ & & & & 0.2607 \\
\hline 0 & $10(40)$ & $11(44)$ & $16(64)$ & \\
\hline 1 & $11(44)$ & $10(40)$ & $4(16)$ & \\
\hline$\geq 2$ & $4(16)$ & $4(16)$ & $5(20)$ & \\
\hline Gestational age, median (Q1, Q3), weeks & & $6(6,6)$ & $39.14(38.79,39.79)$ & $<0.001 * * *$ \\
\hline
\end{tabular}

Comparisons of characteristics among non-pregnant, early-pregnant, and late-pregnant women were assessed using Kruskal-Wallis tests or oneway ANOVA or Mann-Whitney test for continuous variables and chi-squared tests were used for categorical variables. $P$-values less than 0.05 $(P<0.05)$ were considered significant. $* P<0.05$ and $* * * P<0.001$. BMI body mass index, $S D$ standard deviation, $Q 1$ the first quartile, $Q 3$ the third quartile

The analysis of ILC subsets using flow cytometry demonstrated that ILC2s accounted for the majority of total circulating ILCs $\left(\mathrm{CD} 45^{+} \mathrm{Lin}^{-} \mathrm{CD} 127^{+}\right)$in late-pregnant women while occupied smaller parts in early-pregnant $(P<0.001)$ and non-pregnant women $(P<0.001)$ (Fig. 2a). Furthermore, the percentage of ILC2 in total ILCs in early-pregnant women was statistically higher than non-pregnant women $(P=0.0364)$ (Fig. 2a and b). ILC2 proportion in circulating $\mathrm{CD} 45^{+}$cells shared the same variation trend as that in total ILCs that the ILC2 percentage was much higher in latepregnant women than that in early-pregnant $(P=0.0087)$ and non-pregnant women $(P<0.001)$, while the subtle difference was found between early-pregnant and non-pregnant women $(P=0.0305)$ (Fig. 2c). We further evaluated the absolute counts of ILC2s per milliliter of blood in three study groups. It turned out that the count of ILC2 per milliliter of blood in late-pregnant women was statistically higher than that in early-pregnant $(P=0.0403)$ and non-pregnant women $(P<0.001)$ (Fig. 2d). Although there was no significant difference in circulating ILC2 counts between early-pregnant and non-pregnant women $(P>0.05)$, the increasing trend still existed.

Circulating ILC3s in late-pregnant women accounted for a much smaller part of total ILCs than early-pregnant $(P<0.001)$ and non-pregnant women $(P<0.001)$, statistical difference was also observed between early-pregnant and non-pregnant women $(P<0.001)$ (Fig. 2a and b). The absolute count of ILC3s per milliliter of blood and the percentage in $\mathrm{CD}_{4} 5^{+}$cells were much lower in late-pregnant women than those in early-pregnant $(P=0.0016 ; P=0.0021)$ and non-pregnant women $(P=0.0026 ; P=0.0105)$, whereas there was no difference between non-pregnant and earlypregnant women (both $P>0.05$ ) (Fig. $2 \mathrm{c}$ and d). Although, same as ILC3s, circulating ILC1s in late-pregnant women accounted for a smaller proportion of total ILCs than early-pregnant $(P<0.001)$ as well as non-pregnant women $(P<0.001)$ (Fig. 2a and b). There was no difference found in neither ILC1 percentages in $\mathrm{CD} 45^{+}$cells nor absolute cell counts among the three study groups (Fig. $2 \mathrm{c}$ and d).

Taken together, these results reveal the dynamic changes of ILC subset distribution during pregnancy and suggest that these changes are associated with different trimesters of pregnancy.

\section{Elevating Estradiol and Progesterone Levels During Pregnancy Are Correlated with the Increasing Level of Circulating ILC2s}

Next, we evaluated plasma estradiol and progesterone levels of non-pregnant and pregnant women of different trimesters. The estradiol and progesterone levels were low in non-pregnant women and increased gradually as pregnancy proceeded and doubled in late-pregnant women as of early-pregnant women $(P<0.001)$, as previously reported $[24,29]$. The estradiol level in early-pregnant women was significantly higher than non-pregnant women $(P<0.001)$, while the progesterone level varied slightly $(P=0.0418)$ (Fig. 3a and b). Increasing estradiol and progesterone level shared the same variation trend 


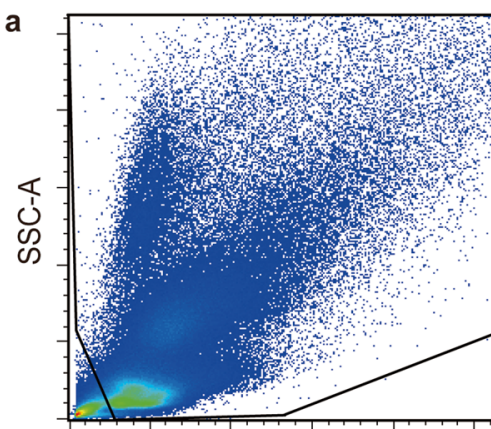

FSC-A

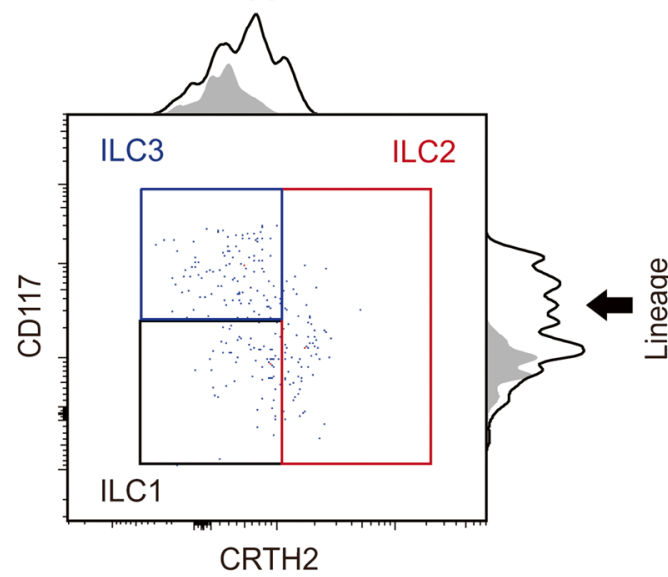

b

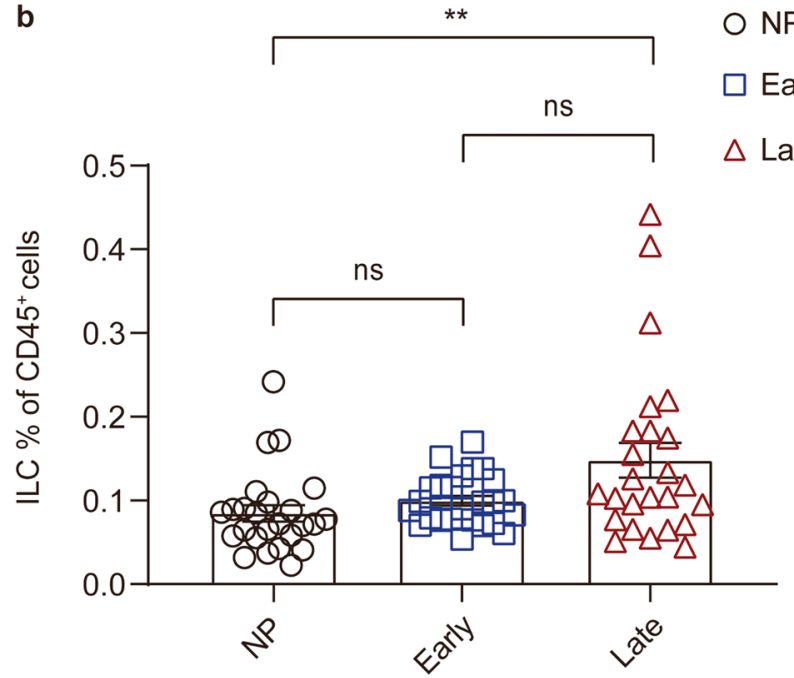

Fig. 1 Late pregnancy displays the highest circulating ILC proportion in human peripheral blood. a Flow cytometry gating strategies for the identification of peripheral blood ILC subsets. ILCs are defined as $\mathrm{CD}_{4} 5^{+} \mathrm{Lin}^{-}$(including CD3e, CD4, CD5, CD14, CD19, CD56, CD11c, CD11b, CD34, CD123, TCRa $\beta$, TCR $\gamma \delta$, and FceRIa) $\mathrm{CD} 127^{+}$cells. The distribution of ILC1 (CD117 ${ }^{-} \mathrm{CRTH}^{-}$; black), ILC2 $\left(\mathrm{CRTH} 2^{+}\right.$; red) and ILC3s $\left(\mathrm{CD} 117^{+} \mathrm{CRTH}^{-}\right.$; blue) were deter-

with circulating ILC2s, which also reached peak levels in late-pregnant women (Table 2). Since previous studies show that sex hormones have a positive regulatory effect on ILC2s

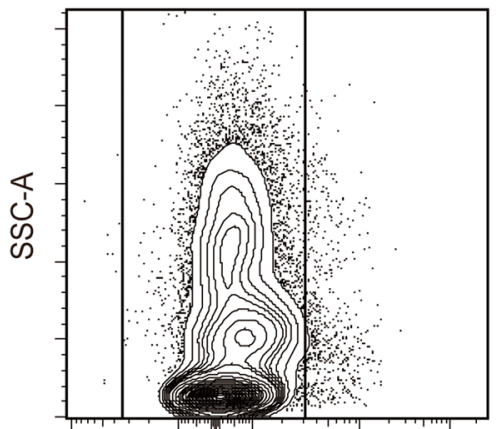

Live/dead dye

FSC-A

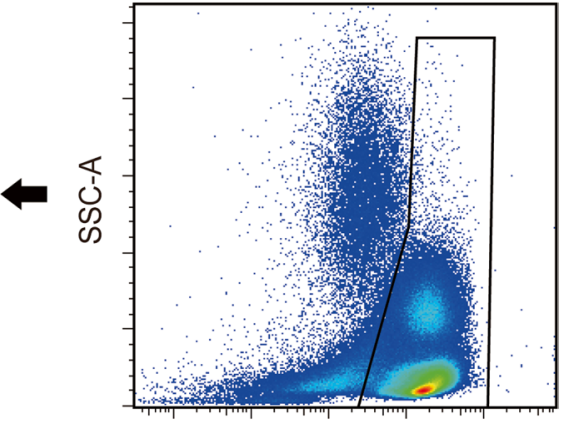

CD45

CD127

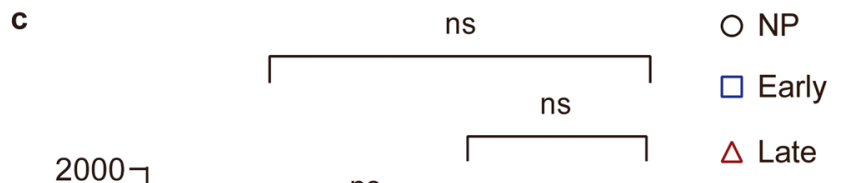

mined. Histograms represent expression analysis with the FMO as grey area and the stained sample as line. Comparison of circulating ILC percentage in $\mathrm{CD}_{4} 5^{+}$cells (b) and absolute counts (c) in nonpregnant, early-pregnant, and late-pregnant women. $N P$, non-pregnant women. Data are shown as means \pm SEMs and were analyzed by Kruskal-Wallis tests. Each point indicates an individual. $* * P<0.01$. $n s$, not significant

in murine uterus [43], to further understand the association of sex hormone level with ILC2s in human peripheral blood, we employed the Spearman's correlation analysis and found 
Reproductive Sciences (2022) 29:1124-1135

1129

a

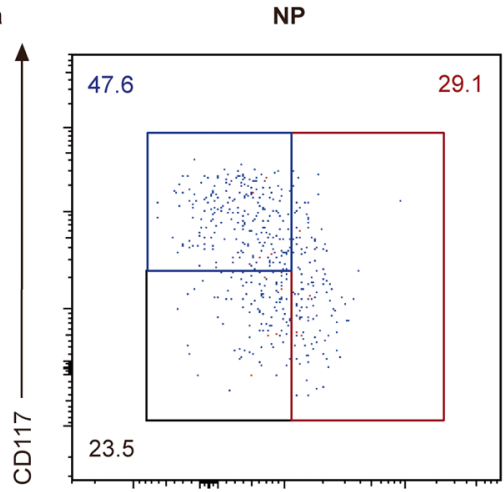

Early

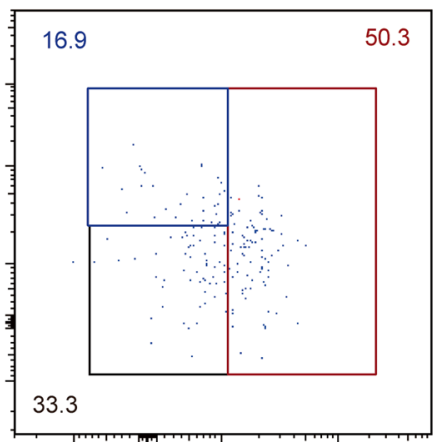

Late

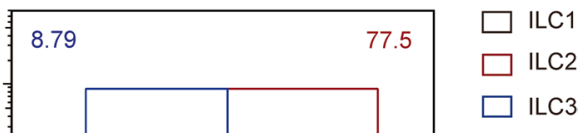

b

CRTH2

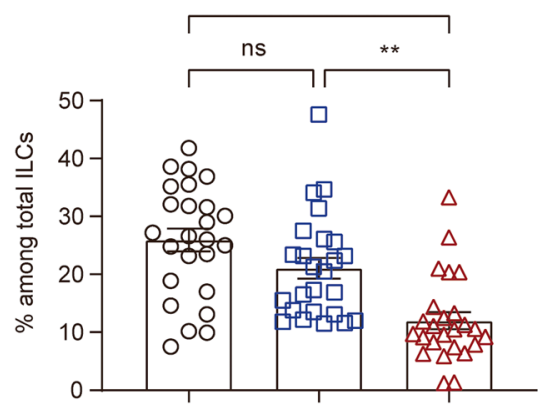

c

ILC1

ILC2

ILC3
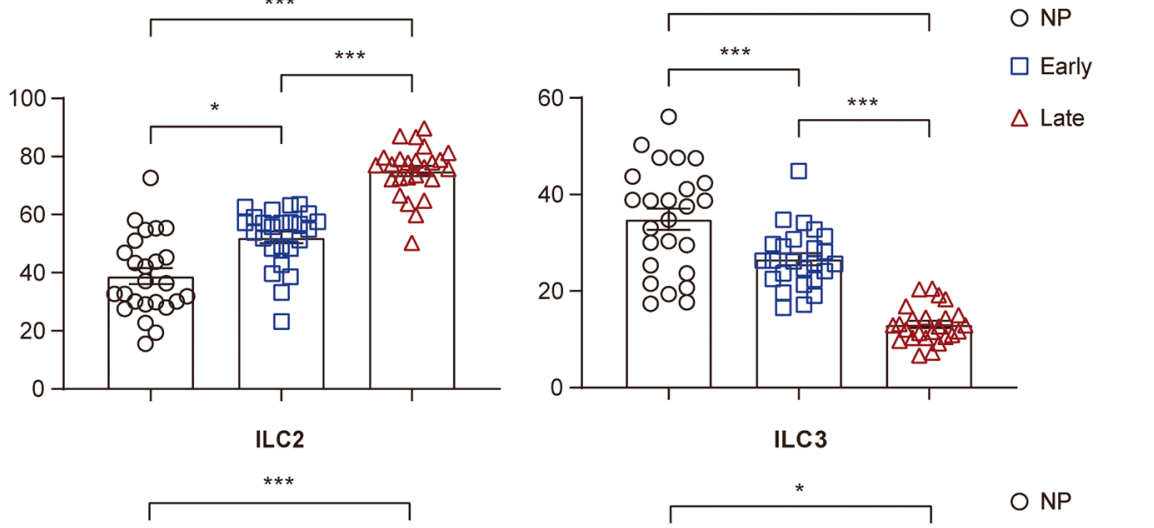

ns
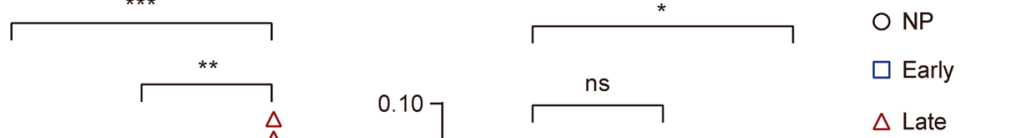

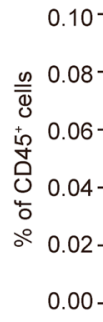
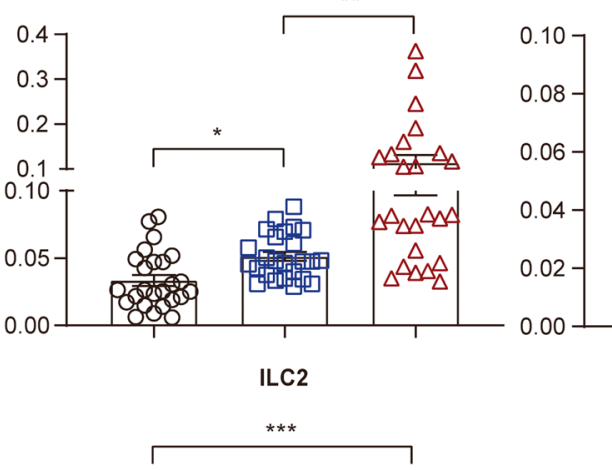

$\infty$

d

ILC1

ILC2
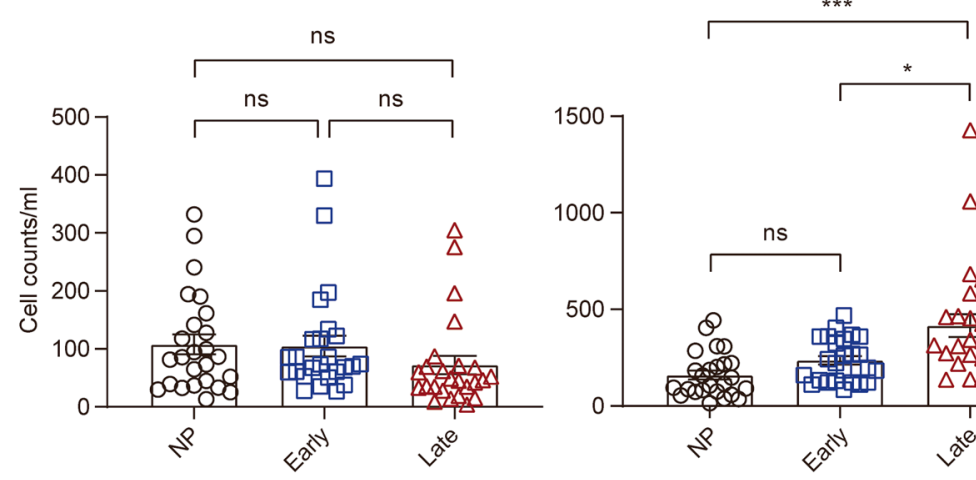

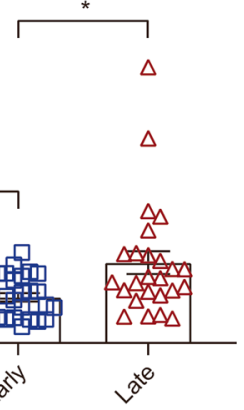

O NP

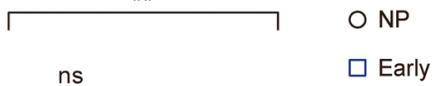

Springer 
4Fig. 2 ILC subsets distribute dynamically in peripheral blood of nonpregnant women and pregnant women of different trimesters. a-d Analysis results for ILC subsets in peripheral blood samples of nonpregnant, early-pregnant, and late-pregnant women. a Representative flow cytometry plots are shown in which numbers indicate the frequency of flow cytometric events. Comparison of proportion of ILC subsets within total ILCs (b), ILC subset percentage in CD45 cells (c) and absolute counts per milliliter of blood (d) in peripheral blood of non-pregnant women (black circles), early-pregnant women (blue squares), and late-pregnant women (red triangles). Data are shown as means \pm SEMs and were analyzed by Kruskal-Wallis tests. Each point indicates an individual. $* P<0.05$, $* * P<0.01$, and $* * * P<0.001 . n s$, not significant suggest that the dynamical distribution of circulating ILC subsets may be a result of physiological changes of plasma estradiol and progesterone level during pregnancy.

\section{Estrogen and Progesterone Receptors in Circulating ILC2s Change Differently Before and During Pregnancy}

Sex hormones regulate physiological processes via their receptors. To further clarify the association of plasma
Fig. 3 Plasma estradiol and progesterone levels increase as pregnancy proceeds. Comparison of concentrations of estradiol (a) and progesterone (b) in plasma of non-pregnant women (black circles), early-pregnant women (blue squares), and latepregnant women (red triangles). Data are shown as means \pm SEMs and were analyzed by Kruskal-Wallis tests. $* P<0.05$ and $* * * P<0.001$
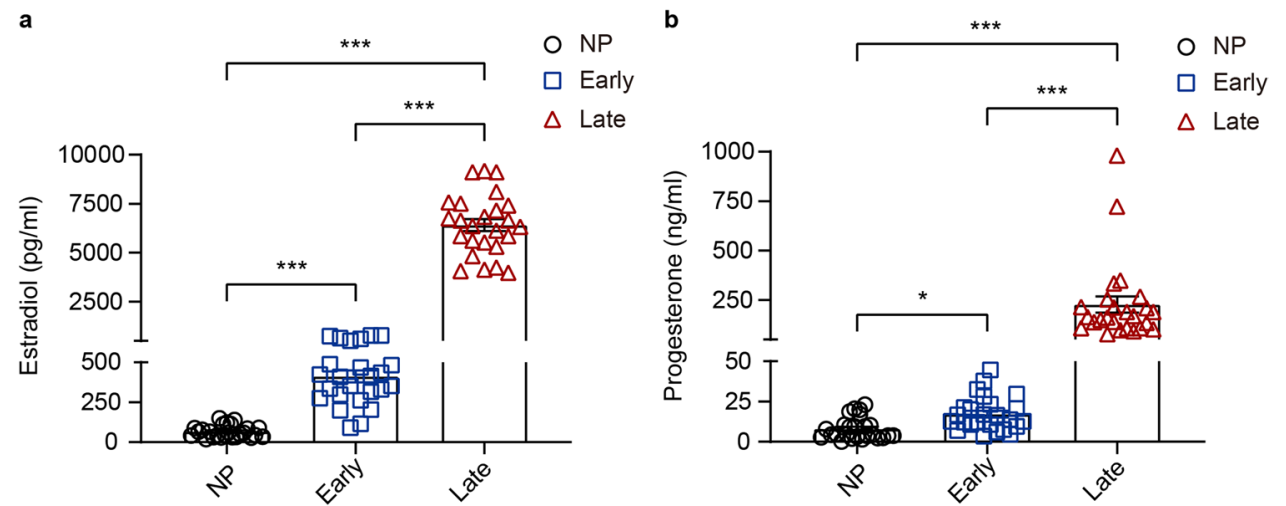

Table 2 Levels of circulating ILC2, estradiol, and progesterone

\begin{tabular}{lllll}
\hline & Non-pregnant & Early-pregnant & Late-pregnant & $P$ value \\
\hline$N$ & 25 & 25 & 25 & \\
ILC2 & & & & \\
$\quad$ Percentage in ILCs, $\%$ & $36.40(29.90,46.90)$ & $55.80(48.30,57.50)$ & $77(72.20,79.10)$ & $<0.001 * * *$ \\
$\quad$ Percentage in CD45 ${ }^{+}$cells, $\%$ & $0.02(0.02,0.04)$ & $0.04(0.03,0.06)$ & $0.07(0.05,0.12)$ & $<0.001 * * *$ \\
$\quad$ Absolute count, per ml & $98.81(64.02,186.15)$ & $191.22(113.02,298.87)$ & $289.61(229.21,397.14)$ & $<0.001 * * *$ \\
Estradiol, pg/ml & $57.10(34.16,88.56)$ & $408.48(297.93,488.08)$ & $6362.75(5534.62,7410.15)$ & $<0.001 * * *$ \\
Progesterone, $\mathrm{ng} / \mathrm{ml}$ & $4.61(3.24,10.22)$ & $13.77(10.70,21.26)$ & $164.37(108.61,214.33)$ & $<0.001 * * *$ \\
\hline
\end{tabular}

Variables are shown as median $(\mathrm{Q} 1, \mathrm{Q} 3)$. Comparisons of levels of circulating ILC2s, plasma estradiol, and progesterone among non-pregnant, early-pregnant, and late-pregnant women were assessed using Kruskal-Wallis tests. $P$-values less than $0.05(P<0.05)$ were considered significant. $* * * P<0.001$. $Q 1$ the first quartile, $Q 3$ the third quartile

that the proportion of circulating ILC2s either in total ILCs or in $\mathrm{CD} 45^{+}$cells was positively correlated both with estradiol $(P<0.001)$ and progesterone $(P<0.001)$ (Table 3$)$. Our results

Table 3 Spearman correlations among circulating ILC2, estradiol, and progesterone

\begin{tabular}{lll}
\hline & Estradiol & Progesterone \\
\hline Percentage in ILCs & $0.7933^{* * *}$ & $0.6752^{* * * *}$ \\
Percentage in CD45 ${ }^{+}$cells & $0.5876^{* * *}$ & $0.4620^{* * * *}$ \\
Absolute count per ml & $0.5915^{* * *}$ & $0.4638^{* * *}$ \\
\hline
\end{tabular}

$* * *$ Correlation is significant at the 0.001 level (2-tailed) estradiol and progesterone with circulating ILC2s, we randomly chose six women in each study group and analyzed the ER $\alpha$, which is highly expressed in ILC2s of murine uterus [43] and progesterone receptor (PR) levels in ILC2s (Table S1). We found that in peripheral blood, ILC2s expressing ER $\alpha$ were also $\mathrm{PR}^{+}$(Fig. 4a). Notably, the percentage of $\mathrm{ER} \alpha^{+}$ILC2 in late-pregnant women was significantly higher than early-pregnant $(P=0.0120)$ and non-pregnant women $(P<0.001)$, while no statistical difference was found between early-pregnant and non-pregnant women $(P>0.05)$ (Fig. $4 \mathrm{a}$ and $\mathrm{b})$. In addition, a higher percentage of $\mathrm{PR}^{+}$ILC2 was observed in 


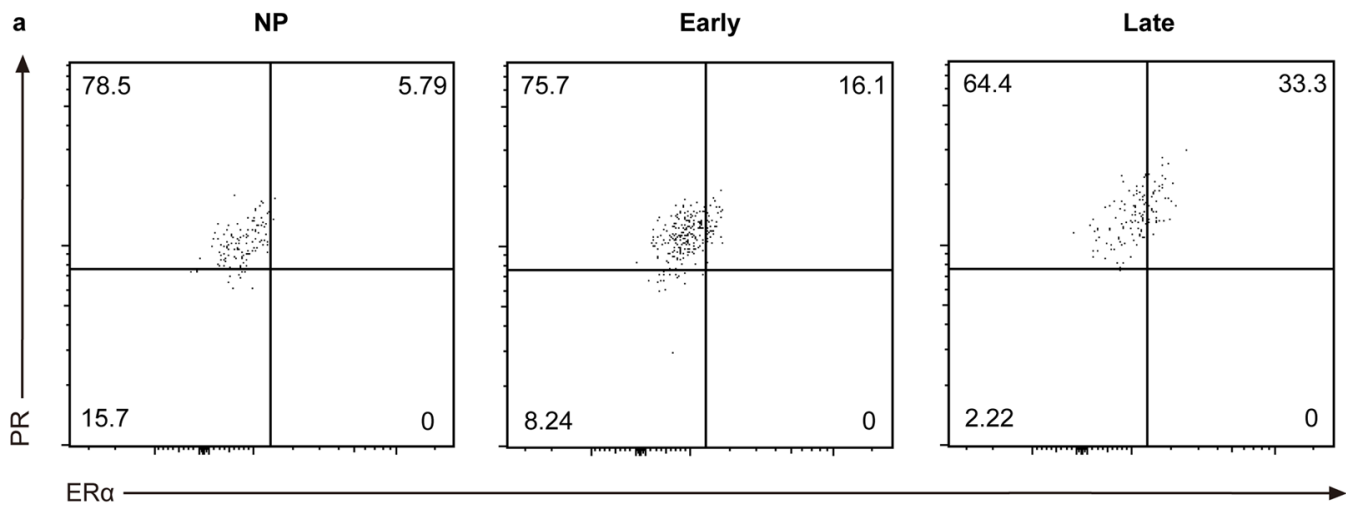

b
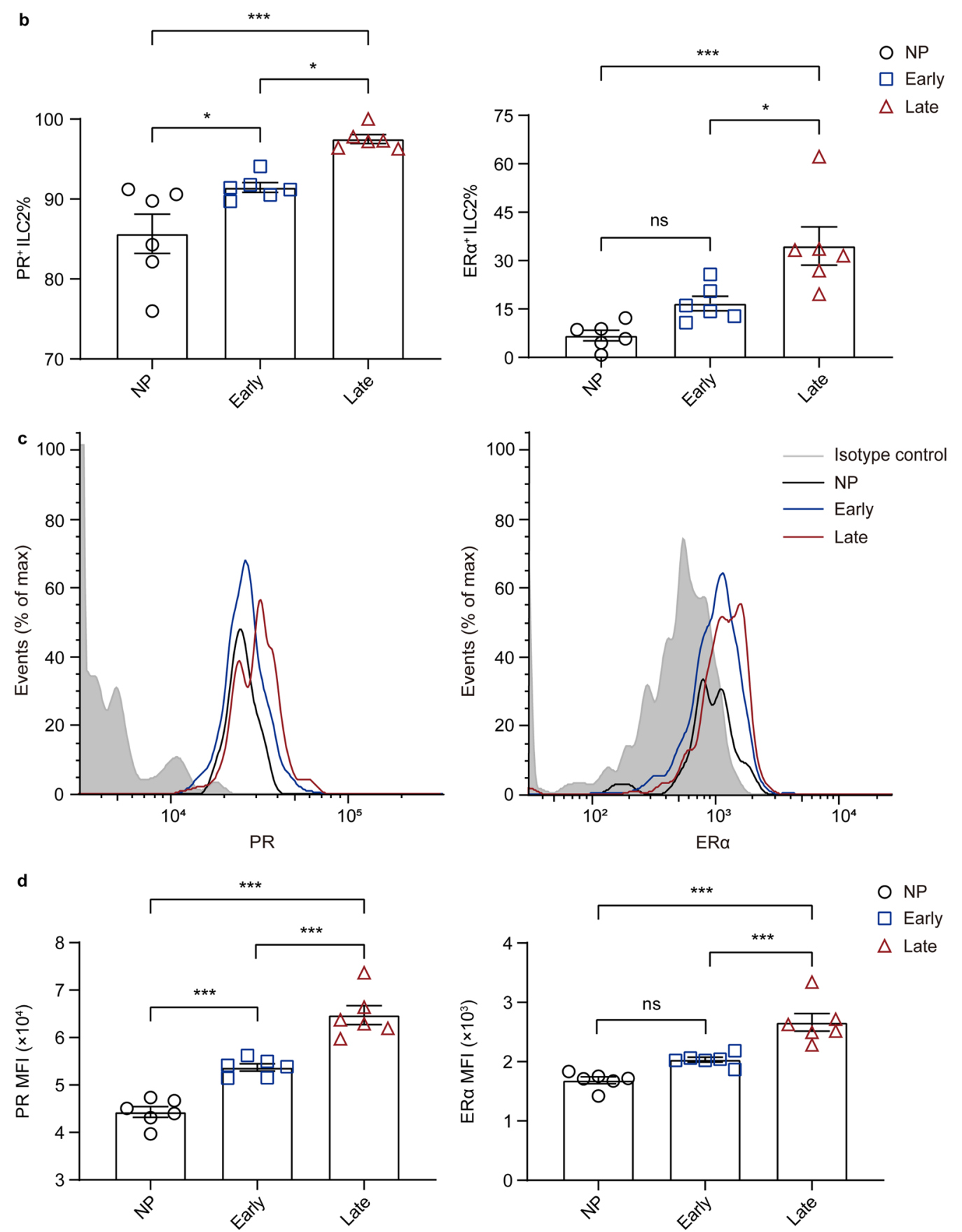
४Fig. 4 The estrogen and progesterone receptors in circulating ILC2s change differently in non-pregnant and pregnant women of different trimesters. a-d Flow cytometric analysis for PR and ER $\alpha$ in peripheral ILC2s of non-pregnant, early-pregnant, and late-pregnant women. a Representative flow cytometry plots are shown in which numbers indicate the frequency of flow cytometric events. b Comparison of and $\mathrm{PR}^{+} \mathrm{ILC} 2$ and $\mathrm{ER} \alpha^{+} \mathrm{ILC} 2$ proportion in peripheral blood of non-pregnant women (black circles), early-pregnant women (blue squares), and late-pregnant women (red triangles). c Representative flow cytometry histograms of intracellular PR and ER $\alpha$ in circulating ILC2s from isotype control (grey area), non-pregnant women (black lines), early-pregnant women (blue lines), and late-pregnant women (red lines). d Quantification of PR and ER $\alpha$ mean fluorescence intensity (MFI) of circulating ILC2s. Data are shown as means \pm SEMs and were analyzed by one-way ANOVA. $* P<0.05$ and $* * * P<0.001$. $n s$, not significant

late-pregnant women than early-pregnant $(P=0.0297)$ and non-pregnant women $(P<0.001)$, and the ratio differed slightly between early-pregnant and non-pregnant women $(P=0.0380)$ (Fig. 4a and b). Furthermore, examination of intracellular PR and ER $\alpha$ expression showed patterns of differential expression in ILC2s, in which PR and ER $\alpha$ expression by late-pregnant samples was much higher to that by non-pregnant and early-pregnant samples (both $P<0.001$ ) (Fig. 4c and d). The observation of differential hormone receptor expression in circulating ILC2s among non-pregnant, early-pregnant, and late-pregnant women indicates the association of estradiol and progesterone with ILC2s in human peripheral blood.

\section{Discussion}

ILCs have been reported as a new population of innate immune cells and regarded as the innate equivalents of $\mathrm{T}$ cells $[13,48]$. Emerging from the lymphoid lineage but do not express antigen-specific receptors or undergo clonal selection [49], ILCs react rapidly to signals from injured sites and produce corresponding cytokines, which eventually lead to multiple immune pathways. According to the diverse expression of transcriptional factors, helper ILCs have been divided into three subsets - ILC1s, ILC2s, and ILC $3 \mathrm{~s}$, which are counterparts of T helper cells. ILCs are widely distributed in mucosal and non-mucosal tissues, including the skin, intestines, lungs, spleen, and secondary lymphoid tissues $[13,48]$. Here we analyzed the proportion of total ILCs and each ILC subset in total ILCs in peripheral blood of non-pregnant and pregnant women of different trimesters and demonstrated that the proportion of ILCs in peripheral blood of late-pregnant women was statistically higher than early-pregnant and non-pregnant women. Furthermore, the proportion and absolute counts of circulating ILC2s were significantly higher in late-pregnant women than early-pregnant and non-pregnant women, while ILC3s were much lower. Compared to non-pregnant women, the proportion of circulating ILC2s in early-pregnant women increased slightly, and no difference was found in the proportion of ILC3s. These results appear to align with previous reports about human decidua [12, 16, 19]. Additionally, we found that the middle trimester seemed to be the transitional period for dynamical changes of circulating ILC subsets (Table S2, Fig. S1). Based on our results, we hypothesized that helper type ILCs might be involved in maintaining normal pregnancy by changing the distribution pattern in different trimesters.

Sex hormones are critical to a healthy pregnancy and can modulate the distribution of immune cells. To determine the factors that contributed to the dynamic distribution of ILC subsets in peripheral blood of non-pregnant and pregnant women of different trimesters, we detected plasma estradiol and progesterone levels. Agreed with previous studies, we showed that the levels of plasma estradiol and progesterone were increased along with pregnancy proceeds and reached peak value during late pregnancy [24, 25], which was consisted with the variation trend of circulating ILC2s. Spearman's correlation analysis demonstrated positive correlation between sex hormone levels and ILC2 proportion, which suggests that dynamical distribution of circulating ILC subsets during pregnancy may be a result of physiological elevating of plasma estradiol and progesterone level. The higher expression of estrogen and progesterone receptors in circulating ILC2s of late-pregnant women provide prima facie evidence for our hypothesis. Being well studied, estradiol in human and mice is recognized to regulate both adaptive and innate immune cells [24-28]. A recent study has shown that ILC2s in murine uterus can be regulated by estradiol through $\mathrm{ER} \alpha$ [31]. Another previous research of murine ILC2s in different tissues indicates that ILC2s express a narrow panel of sex hormone receptors while progesterone receptor is barely expressed [37]. In human, progesterone is reported to favor the development of Th cells producing Th2-type cytokines [29], the counterparts of ILC2s. However, the progesterone receptor in human ILC2s is poorly studied. We observed the expression of progesterone receptors in human circulating ILC2s for the first time. Since sex hormone receptors are nuclear transcription factors as well as modulators of cell signaling pathways [25], our results suggest a potential role of plasma sex hormones regulating ILC2s in human peripheral blood.

In view of the fetus being a semiallograft, maintaining the balance of immune system is of great importance to a successful and healthy pregnancy. The critical roles of $\mathrm{T}$ cells as well as NK cells in a successful pregnancy have been demonstrated in lots of studies [1, 4, 17, 50], while the exact roles of helper type ILCs during pregnancy remain unknown. There was some research about uterine and decidual ILCs $[8,10,12,16,17,19,22,50]$, but the origin of 
these ILCs is still undetermined. A recent study considers ILCs as tissue-resident immune cells [51], the other one has found that circulating ILCs are immature ILCs [46], and the mechanism under which ILCs circulate between peripheral blood and tissues are still uncertain. Based on the above, we hypothesized that ILCs in peripheral blood may be the source of those in tissues, which needs further investigation. Although ILCs in maternal-fetal compartments have been studied over the years, the distributions of ILC subsets in peripheral blood during pregnancy are barely explored. Our data reveal the dynamic changes and distribution of circulating ILC subsets during normal pregnancy and the underlying role of estradiol and progesterone that modulates the proportion of ILC subsets.

Compared with the first trimester with a high risk of miscarriage [52] and the third trimester, which is about to undergo parturition, the second trimester seems to be a relatively stable state. Therefore, in terms of changes during pregnancy, we mainly focus on early and late pregnancy, which, in our opinion, were the most representative periods and better to show the variation of ILCs in peripheral blood. Furthermore, by detecting estradiol and progesterone receptors on circulating ILC2s in non-pregnant and pregnant women, our study finds a way for sex hormones to influence ILC distribution. However, our study does have some limitations that must be deliberated. The direct evidence of sex hormones regulating ILC subsets was insufficient, in vitro experiments are required. In addition, how circulating helper type ILCs play their roles in pregnancy, for example, effector cytokine secretion, also needs to be further studied, and the distribution pattern during pathological pregnancy is worthy of exploring.

In conclusion, this study demonstrated that in different trimester of normal pregnancy, despite NK cells, circulating helper type ILC subsets distributed diversely, as the level of ILC2s gradually increased along with pregnancy proceeded while ILC3s decreased. Elevating dramatically as pregnancy proceeded, estradiol and progesterone levels agreed with the variation trend of circulating ILC2s and might regulate ILC distribution via their receptors. We hypothesized that the dynamic changes of circulating ILC subsets might be adaptive feedback of pregnancy and are involved in fetal-maternal tolerance. Furthermore, increasing sex hormones may modulate transcriptional activity, thereby influencing the distribution of ILC subsets.

Abbreviations ILCs: Innate lymphoid cells; PBMCs: Peripheral blood mononuclear cells; ILC1: Type 1 innate lymphoid cells; ILC2: Type 2 innate lymphoid cells; ILC3: Type 3 innate lymphoid cells; NK cells: Natural killer cells; Th cells: T helper cells; E1: Estrone; E2: Estradiol; E3: Estriol; PE-Cy7: R phycoerythrin-cyanine 7; PE: Phycoerythrin; ER $\alpha$ : Estrogen receptor $\alpha$;
RIA: Radioimmunoassay; BMI: Body mass index; PR: Progesterone receptor; NP: Non-pregnant women.

Supplementary Information The online version contains supplementary material available at https://doi.org/10.1007/s43032-021-00834-6.

Acknowledgements We are thankful to the participants for the cooperation. We also thank the technologist from the clinical laboratory for their help in collecting samples.

Funding This work was supported by the National Key Research and Development Program of China (2017YFC1001303 to H.H., 2019YFA0802604 to X.C.) and the National Natural Science Foundation of China (31801231 to X.C., 82001649 to H.L.).

Data Availability The data that support the findings of this study are available from the corresponding author upon reasonable request.

\section{Declarations}

Ethics Approval Ethical approval for the study was obtained from the Ethics Review Committee/Institutional Review Board (GKLW 2017-01)

Consent to Participate Informed consent was obtained from all individual participants included in the study.

Consent for Publication The participant has consented to the submission of the research article to the journal.

Conflict of Interest The authors declare no competing interests.

Open Access This article is licensed under a Creative Commons Attribution 4.0 International License, which permits use, sharing, adaptation, distribution and reproduction in any medium or format, as long as you give appropriate credit to the original author(s) and the source, provide a link to the Creative Commons licence, and indicate if changes were made. The images or other third party material in this article are included in the article's Creative Commons licence, unless indicated otherwise in a credit line to the material. If material is not included in the article's Creative Commons licence and your intended use is not permitted by statutory regulation or exceeds the permitted use, you will need to obtain permission directly from the copyright holder. To view a copy of this licence, visit http://creativecommons.org/licenses/by/4.0/.

\section{References}

1. Ghaebi M, Nouri M, Ghasemzadeh A, Farzadi L, Jadidi-Niaragh $\mathrm{F}$, Ahmadi M, et al. Immune regulatory network in successful pregnancy and reproductive failures. Biomed Pharmacother. 2017;88:61-73. https://doi.org/10.1016/j.biopha.2017.01.016.

2. PrabhuDas M, Bonney E, Caron K, Dey S, Erlebacher A, Fazleabas A, et al. Immune mechanisms at the maternal-fetal interface: perspectives and challenges. Nat Immunol. 2015;16(4):328-34. https://doi.org/10.1038/ni.3131.

3. Erlebacher A. Immunology of the maternal-fetal interface. Annu Rev Immunol. 2013;31:387-411. https://doi.org/10.1146/annur ev-immunol-032712-100003. 
4. Figueiredo AS, Schumacher A. The T helper type 17/regulatory $\mathrm{T}$ cell paradigm in pregnancy. Immunology. 2016;148(1):13-21. https://doi.org/10.1111/imm.12595.

5. Wang SC, Li YH, Piao HL, Hong XW, Zhang D, Xu YY, et al. PD-1 and Tim-3 pathways are associated with regulatory CD8+ T-cell function in decidua and maintenance of normal pregnancy. Cell Death Dis. 2015;6(5):e1738. https://doi.org/10.1038/cddis. 2015.112.

6. Miller D, Gershater M, Slutsky R, Romero R, Gomez-Lopez N. Maternal and fetal $\mathrm{T}$ cells in term pregnancy and preterm labor. Cell Mol Immunol. 2020;17(7):693-704. https://doi.org/10.1038/ s41423-020-0471-2.

7. Aluvihare VR, Kallikourdis M, Betz AG. Regulatory T cells mediate maternal tolerance to the fetus. Nat Immunol. 2004;5(3):26671. https://doi.org/10.1038/ni1037.

8. Miller D, Motomura K, Garcia-Flores V, Romero R, GomezLopez N. Innate lymphoid cells in the maternal and fetal compartments. Front Immunol. 2018;9:2396. https://doi.org/10.3389/ fimmu.2018.02396.

9. Negishi Y, Takahashi H, Kuwabara Y, Takeshita T. Innate immune cells in reproduction. J Obstet Gynaecol Res. 2018;44(11):202536. https://doi.org/10.1111/jog.13759.

10. Vacca P, Vitale C, Munari E, Cassatella MA, Mingari MC, Moretta L. Human innate lymphoid cells: their functional and cellular interactions in decidua. Front Immunol. 2018;9:1897. https://doi.org/10.3389/fimmu.2018.01897.

11. Li Y, Zhang J, Zhang D, Hong X, Tao Y, Wang S, et al. Tim-3 signaling in peripheral NK cells promotes maternal-fetal immune tolerance and alleviates pregnancy loss. Sci Signal. 2017;10(498). https://doi.org/10.1126/scisignal.aah4323.

12. Vacca P, Montaldo E, Croxatto D, Loiacono F, Canegallo F, Venturini PL, et al. Identification of diverse innate lymphoid cells in human decidua. Mucosal Immunol. 2015;8(2):254-64. https://doi. org/10.1038/mi.2014.63.

13. Eberl G, Colonna M, Di Santo JP, McKenzie AN. Innate lymphoid cells. Innate lymphoid cells: a new paradigm in immunology. Science. 2015;348(6237):aaa6566. https://doi.org/10.1126/science. aaa6566.

14. Klose CS, Artis D. Innate lymphoid cells as regulators of immunity, inflammation and tissue homeostasis. Nat Immunol. 2016;17(7):765-74. https://doi.org/10.1038/ni.3489.

15. Mjösberg JM, Trifari S, Crellin NK, Peters CP, van Drunen CM, Piet B, et al. Human IL-25- and IL-33-responsive type 2 innate lymphoid cells are defined by expression of CRTH2 and CD161. Nat Immunol. 2011;12(11):1055-62. https://doi.org/10.1038/ni. 2104.

16. Doisne JM, Balmas E, Boulenouar S, Gaynor LM, Kieckbusch $\mathrm{J}$, Gardner L, et al. Composition, development, and function of uterine innate lymphoid cells. J Immunol. 2015;195(8):3937-45. https://doi.org/10.4049/jimmunol.1500689.

17. Male V, Hughes T, McClory S, Colucci F, Caligiuri MA, Moffett A. Immature NK cells, capable of producing IL-22, are present in human uterine mucosa. J Immunol. 2010;185(7):3913-8. https:// doi.org/10.4049/jimmunol.1001637.

18. Croxatto D, Micheletti A, Montaldo E, Orecchia P, Loiacono F, Canegallo F, et al. Group 3 innate lymphoid cells regulate neutrophil migration and function in human decidua. Mucosal Immunol. 2016;9(6):1372-83. https://doi.org/10.1038/mi.2016.10.

19. Xu Y, Romero R, Miller D, Silva P, Panaitescu B, Theis KR, et al. Innate lymphoid cells at the human maternal-fetal interface in spontaneous preterm labor. Am J Reprod Immunol. 2018;79(6):e12820. https://doi.org/10.1111/aji.12820.

20. Balmas E, Rana BMJ, Hamilton RS, Shreeve N, Kieckbusch J, Aye I et al. Maternal group 2 innate lymphoid cells control fetal growth and protect from endotoxin-induced abortion in mice. bioRxiv. 2018:348755. https://doi.org/10.1101/348755.
21. Jabrane-Ferrat N, Siewiera J. The up side of decidual natural killer cells: new developments in immunology of pregnancy. Immunology. 2014;141(4):490-7. https://doi.org/10.1111/imm.12218.

22. Li M, Gao Y, Yong L, Huang D, Shen J, Liu M, et al. Molecular signature and functional analysis of uterine ILCs in mouse pregnancy. J Reprod Immunol. 2017;123:48-57. https://doi.org/10. 1016/j.jri.2017.09.003.

23. Mendes J, Rodrigues-Santos P, Areia AL, Almeida J-S, Alves $\mathrm{V}$, Santos-Rosa M, et al. Type 2 and type 3 innate lymphoid cells at the maternal-fetal interface: implications in preterm birth. BMC Immunol. 2021;22(1):28. https://doi.org/10.1186/ s12865-021-00423-x.

24. Kodogo V, Azibani F, Sliwa K. Role of pregnancy hormones and hormonal interaction on the maternal cardiovascular system: a literature review. Clin Res Cardiol. 2019;108(8):831-46. https:// doi.org/10.1007/s00392-019-01441-x.

25. Noyola-Martinez N, Halhali A, Barrera D. Steroid hormones and pregnancy. Gynecol Endocrinol. 2019;35(5):376-84. https://doi. org/10.1080/09513590.2018.1564742.

26. Chen JZ, Wong MH, Brennecke SP, Keogh RJ. The effects of human chorionic gonadotrophin, progesterone and oestradiol on trophoblast function. Mol Cell Endocrinol. 2011;342(1-2):73-80. https://doi.org/10.1016/j.mce.2011.05.034.

27. Berkane N, Liere P, Oudinet JP, Hertig A, Lefevre G, Pluchino $\mathrm{N}$, et al. From pregnancy to preeclampsia: a key role for estrogens. Endocr Rev. 2017;38(2):123-44. https://doi.org/10.1210/ er.2016-1065.

28. Geyer J, Bakhaus K, Bernhardt R, Blaschka C, Dezhkam Y, Fietz D, et al. The role of sulfated steroid hormones in reproductive processes. J Steroid Biochem Mol Biol. 2017;172:207-21. https://doi.org/10.1016/j.jsbmb.2016.07.002.

29. Szekeres-Bartho J, Halasz M, Palkovics T. Progesterone in pregnancy; receptor-ligand interaction and signaling pathways. J Reprod Immunol. 2009;83(1-2):60-4. https://doi.org/10.1016/j. jri.2009.06.262.

30. Nilsson S, Mäkelä S, Treuter E, Tujague M, Thomsen J, Andersson $\mathrm{G}$, et al. Mechanisms of estrogen action. Physiol Rev. 2001;81(4):1535-65. https://doi.org/10.1152/physrev.2001. 81.4 .1535 .

31. Nair RR, Verma P, Singh K. Immune-endocrine crosstalk during pregnancy. Gen Comp Endocrinol. 2017;242:18-23. https://doi org/10.1016/j.ygcen.2016.03.003.

32. Klein SL, Flanagan KL. Sex differences in immune responses. Nat Rev Immunol. 2016;16(10):626-38. https://doi.org/10. 1038/nri.2016.90.

33. Jaillon S, Berthenet K, Garlanda C. Sexual dimorphism in innate immunity. Clin Rev Allergy Immunol. 2019;56(3):30821. https://doi.org/10.1007/s12016-017-8648-x.

34. Kovats S. Estrogen receptors regulate innate immune cells and signaling pathways. Cell Immunol. 2015;294(2):63-9. https:// doi.org/10.1016/j.cellimm.2015.01.018.

35. Seillet $\mathrm{C}$, Laffont $\mathrm{S}$, Trémollières $\mathrm{F}$, Rouquié $\mathrm{N}$, Ribot $\mathrm{C}$, Arnal $\mathrm{J}-\mathrm{F}$, et al. The TLR-mediated response of plasmacytoid dendritic cells is positively regulated by estradiol in vivo through cellintrinsic estrogen receptor $\alpha$ signaling. Blood. 2012;119(2):45464. https://doi.org/10.1182/blood-2011-08-371831.

36. Laffont S, Seillet C, Guéry J-C. Estrogen receptor-dependent regulation of dendritic cell development and function. Front Immunol. 2017;8(108). https://doi.org/10.3389/fimmu.2017. 00108.

37. Lélu K, Laffont S, Delpy L, Paulet P-E, Périnat T, Tschanz SA, et al. Estrogen receptor $\alpha$ signaling in T lymphocytes is required for estradiol-mediated inhibition of Th1 and Th17 cell differentiation and protection against experimental autoimmune encephalomyelitis. J Immunol. 2011;187(5):2386. https://doi.org/10.4049/ jimmunol.1101578. 
38. Mohammad I, Starskaia I, Nagy T, Guo J, Yatkin E, Väänänen K et al. Estrogen receptor $\alpha$ contributes to T cell-mediated autoimmune inflammation by promoting $\mathrm{T}$ cell activation and proliferation. Sci Signal 2018;11(526):eaap9415. https://doi.org/10.1126/ scisignal.aap9415.

39. Calippe B, Douin-Echinard V, Delpy L, Laffargue M, Lélu K, Krust A, et al. 17 $\beta$-estradiol promotes TLR4-triggered proinflammatory mediator production through direct estrogen receptor $\alpha$ signaling in macrophages in vivo. J Immunol. 2010;185(2):1169. https://doi.org/10.4049/jimmunol.0902383.

40. Piccinni MP, Giudizi MG, Biagiotti R, Beloni L, Giannarini L, Sampognaro S, et al. Progesterone favors the development of human T helper cells producing Th2-type cytokines and promotes both IL-4 production and membrane CD30 expression in established Th1 cell clones. J Immunol. 1995;155(1):128.

41. Blanquart E, Laffont S, Guéry J-C. Sex hormone regulation of innate lymphoid cells. Biom J. 2020. https://doi.org/10.1016/j.bj. 2020.11.007.

42. Barkhem T, Carlsson B, Nilsson Y, Enmark E, Gustafsson J, Nilsson S. Differential response of estrogen receptor alpha and estrogen receptor beta to partial estrogen agonists/antagonists. Mol Pharmacol. 1998;54(1):105-12. https://doi.org/10.1124/mol.54.1. 105.

43. Bartemes K, Chen CC, Iijima K, Drake L, Kita H. IL-33-responsive group 2 innate lymphoid cells are regulated by female sex hormones in the uterus. J Immunol. 2018;200(1):229-36. https:// doi.org/10.4049/jimmunol.1602085.

44. Li Q, Liu M, Fu R, Cao Q, Wang Y, Han S, et al. Alteration of circulating innate lymphoid cells in patients with atherosclerotic cerebral infarction. Am J Transl Res. 2018;10(12):4322-30.

45. Perry JS, Han S, Xu Q, Herman ML, Kennedy LB, Csako G et al. Inhibition of LTi cell development by CD25 blockade is associated with decreased intrathecal inflammation in multiple sclerosis.
Sci Transl Med 2012;4(145):145ra06. https://doi.org/10.1126/scitr anslmed.3004140.

46. Lim AI, Li Y, Lopez-Lastra S, Stadhouders R, Paul F, Casrouge A, et al. Systemic human ILC precursors provide a substrate for tissue ILC differentiation. Cell. 2017;168(6):1086-100 e10. https:// doi.org/10.1016/j.cell.2017.02.021.

47. Spits H, Artis D, Colonna M, Diefenbach A, Di Santo JP, Eberl $\mathrm{G}$, et al. Innate lymphoid cells--a proposal for uniform nomenclature. Nat Rev Immunol. 2013;13(2):145-9. https://doi.org/10. 1038/nri3365.

48. Vivier E, Artis D, Colonna M, Diefenbach A, Di Santo JP, Eberl G, et al. Innate lymphoid cells: 10 years on. Cell. 2018;174(5):1054 66. https://doi.org/10.1016/j.cell.2018.07.017.

49. Cherrier DE, Serafini N, Di Santo JP. Innate lymphoid cell development: a T cell perspective. Immunity. 2018;48(6):1091-103. https://doi.org/10.1016/j.immuni.2018.05.010.

50. Le Bouteiller P, Piccinni MP. Human NK cells in pregnant uterus: why there? Am J Reprod Immunol. 2008;59(5):401-6. https://doi. org/10.1111/j.1600-0897.2008.00597.x.

51. Gasteiger G, Fan X, Dikiy S, Lee SY, Rudensky AY. Tissue residency of innate lymphoid cells in lymphoid and nonlymphoid organs. Science. 2015;350(6263):981-5. https://doi.org/10.1126/ science.aac9593.

52. Ammon Avalos L, Galindo C, Li D-K. A systematic review to calculate background miscarriage rates using life table analysis. Birth Defects Res A Clin Mol Teratol. 2012;94(6):417-23. https:// doi.org/10.1002/bdra.23014.

Publisher's Note Springer Nature remains neutral with regard to jurisdictional claims in published maps and institutional affiliations. 